\title{
Electromagnetic fields and nanomagnetic particles increase the osteogenic differentiation of human bone marrow-derived mesenchymal stem cells
}

\author{
MIN-OK KIM ${ }^{1}$, HYUN JUNG $^{2}$, SOO-CHAN KIM ${ }^{3}$, JUNG-KEUG PARK ${ }^{1}$ and YOUNG-KWON SEO ${ }^{1}$ \\ Departments of ${ }^{1}$ Medical Biotechnology and ${ }^{2}$ Chemistry, Dongguk University, Seoul 100-715; ${ }^{3}$ Graduate School \\ of Bio and Information Technology, Hankyong National University, Anseong, Kyonggi-do 456-749, Republic of Korea
}

Received March 28, 2014; Accepted October 14, 2014

DOI: $10.3892 /$ ijmm.2014.1978

\begin{abstract}
Human bone marrow-derived mesenchymal stem cells (hBM-MSCs) are widely used in a number of cell therapies and have osteogenic differentiation capacity. Exposure to electromagnetic fields (EMFs) increases the osteogenic differentiation of hBM-MSCs. Nanomagnetic particles (MPs) also promote the differentiation potential of stem cells. In the present study, we investigated the effects of EMFs and MPs on the osteogenic differentiation of hBM-MSCs.hBM-MSCs were treated with $50 \mu \mathrm{g} / \mathrm{ml}$ of $\mathrm{Fe}_{3} \mathrm{O}_{4}$ MPs or exposed to a frequency of $45 \mathrm{~Hz}$ and an intensity of $1 \mathrm{mT}$ EMF twice every $8 \mathrm{~h}$ per day for 7 days. MP incorporation, EMF exposure and MP incorporation with exposure to EMFs did not induce cytotoxic effects. A strong expression of osteogenic markers (osteocalcin, osteopontin and osteonectin) and von Kossa staining intensity was observed in the cells treated with MPs, the cells exposed to EMFs and in the cells treated with MPs and exposed to EMFs compared with the control group, as shown by immunohistochemical staining. Quantitative RT-PCR revealed that the mRNA expression levels of osteoblast markers [osteocalcin, osteopontin, osteonectin, collagen I, collagen III, bone morphogenetic protein 2 (BMP-2), bone sialoprotein (BSP) and runt-related transcription factor 2 (Runx-2)] were markedly increased in the cells treated with MPs and exposed to EMFs. Furthermore, the mRNA expression of calcium channels (CACNA1C, CACNA1E, CACNA1G and CACNA1I) was activated during osteogenic differentiation. The expression levels of osteogenesis-related proteins (BSP, BMP-2, osteopontin and osteonectin) and phosphorylated extracellular signal-regulated
\end{abstract}

Correspondence to: Dr Young-Kwon Seo, Department of Medical Biotechnology, Dongguk University, Pil-dong 3-ga, Chung-gu, Seoul 100-715, Republic of Korea

E-mail: bioseo@dongguk.edu

Key words: electromagnetic fields, osteogenic differentiation, nanomagnetic particles, human bone marrow-derived mesenchymal stem cells kinase (p-ERK) were increased in the cells treated with MPs, those exposed to EMFs and in the cells treated with MPs and exposed to EMFs compared with the control group, as shown by western blot analysis. Fluorescence-activated cell sorting (FACS) analysis was performed for the hBM-MSC markers, CD73, CD90 and CD105. The expression levels of hBM-MSC surface antigens were decreased in the cells treated with MPs, those exposed to EMFs and in the cells treated with MPs and exposed to EMFs compared with the control group. The cell numbers were determined to be approximately $3.4 \times 10^{5}$ cells in the control group, $3.7 \times 10^{5}$ cells in the MP-treated group, $3.1 \times 10^{5}$ cells in the group exposed to EMFs and $3.9 \times 10^{5}$ cells in the group treated with MPs and exposed to EMFs. The cell mitochondrial activity among the 4 experimental groups was similar. The hBM-MSCs treated with MPs and exposed to EMFs showed an increase in alkaline phosphatase (ALP) activity. Taken together, these results suggest that the treatment of hBM-MSCs with MPs or exposure to EMFs increases osteogenic differentiation, and that treatment with MPs in conjunction with EMF exposure is more effective in increasing osteogenic differentiation.

\section{Introduction}

Human mesenchymal stem cells (MSCs) are widely used adult stem cells which have self-renewal potential and multipotent cell differentiation capacity. In particular, human bone marrowderived mesenchymal stem cells (hBM-MSCs) have been used experimentally in a number of cell therapies and in regenerative medicine due to their abundant and non-tumorigenic properties (1). hBM-MSCs have the ability to differentiate into various types of cells, such as fibroblasts, chondrocytes, adipocytes and osteoblasts (2). In addition, hBM-MSCs play an essential role in the repair of bone (3).

Previous studies have indicated that bone regeneration occurs following an electrical stimulation and leads to bone development $(4,5)$. Based on this knowledge, the effect of electromagnetic fields (EMFs) on osteogenesis has been investigated. Recently, it has been reported that an extremely low frequency of EMF stimulation plays an essential role in the osteogenic differentiation and proliferation of hBM-MSCs. Tsai et al (6) demonstrated that exposure to an extremely low 
frequency of EMFs $(7.5 \mathrm{~Hz})$ played a modulatory role in the osteogenic differentiation of hBM-MSCs, with an increase in alkaline phosphatase (ALP) levels. Sun et al (7) demonstrated that a frequency of $15 \mathrm{~Hz}$ EMFs increased the expression of osteogenesis-related genes in hBM-MSCs.

Nanomagnetic particles (MPs) have been widely used in biomedicine. MPs may be applied to apply drug delivery, biological labels and the detection of proteins due to their controllable size and magnetic properties (8). Cartmell et al (9) reported that the mechanical stimulation of primary human osteoblasts by magnetic particle technology affected osteoblastic activity. In the present study, iron oxide $\left(\mathrm{Fe}_{3} \mathrm{O}_{4}\right)$ MPs were encapsulated with a polyethylene glycol (PEG)-phospholipid shell for enhanced biocompatibility.

In the present study, we investigated the effects of EMF exposure and $\mathrm{Fe}_{3} \mathrm{O}_{4} \mathrm{MP}$ treatment on the osteogenic differentiation of hBM-MSCs. hBM-MSCs were treated with $50 \mu \mathrm{g} / \mathrm{ml}$ of $\mathrm{Fe}_{3} \mathrm{O}_{4} \mathrm{MPs}$ or exposed to a frequency of $45 \mathrm{~Hz}$ at an intensity of $1 \mathrm{mT}$ EMF twice every $8 \mathrm{~h}$ per day for 7 days. We also examined whether treatment with $\mathrm{Fe}_{3} \mathrm{O}_{4}$ MPs in conjunction with exposure to EMFs is more effective in enhancing osteogenic differentiation. The osteogenic differentiation was analyzed by immunohistochemical staining, western blot analysis, quantitative reverse transcription-polymerase chain reaction (RT-qPCR) and by measuring ALP activity. Lactate dehydrogenase (LDH) activity in the 4 experimental groups was similar; this suggests that treatment did not affect LDH secretion and did not induce damage to the cell membrane.

\section{Materials and methods}

Cell culture. hBM-MSCs were purchased from Lonza (Basel, Switzerland) and maintained in culture in Dulbecco's modified Eagle's medium (DMEM; Welgene, Daejeon, Korea) supplemented with $10 \%$ fetal bovine serum (FBS; Lonza), $1 \%$ penicillin/streptomycin (PS; Welgene), $25 \mu \mathrm{M}$ L-ascorbic acid 2-phosphate (Sigma-Aldrich, St. Louis, MO, USA) in a $37^{\circ} \mathrm{C}$ incubator in a humidified atmosphere of $5 \% \mathrm{CO}_{2}$. The hBM-MSCs were used from passages 4 to 7 with similar results obtained throughout.

Osteogenic differentiation of $h B M-M S C s$. Osteogenic differentiation medium consisted of DMEM (Welgene) supplemented with $10 \%$ fetal bovine serum (FBS; Lonza), $1 \%$ penicillin/ streptomycin (PS; Welgene), $10 \mathrm{mM} \beta$-glycerophosphate (Sigma-Aldrich), $50 \mu \mathrm{M}$ L-ascorbic acid 2-phosphate (SigmaAldrich) and $100 \mathrm{nM}$ dexamethasone (Sigma-Aldrich). The medium was changed every 2-3 days.

Exposure to EMFs. A schematic representation of the sinusoidal EMF device is presented in Fig. 1. The EMF device was placed in a $37^{\circ} \mathrm{C}$ incubator in a humidified atmosphere of $5 \% \mathrm{CO}_{2}$.

The stimulation unit is designed to handle a pair of identical coils of $30 \mathrm{~cm}$ in diameter assembled in a Helmholtz configuration. The pair of coils operates on alternating current, generating an EMF. The current in the coil is controlled by a generator. The applied magnetic field consisted of $45 \mathrm{~Hz}$ frequency and an intensity of $1 \mathrm{mT}$ twice every $8 \mathrm{~h}$ per day for 7 days. The cells were seeded into a culture plate or dish.
Preparation and characterization of $\mathrm{Fe}_{3} \mathrm{O}_{4} \mathrm{MPs}$. Waterdispersible and biocompatible $\mathrm{Fe}_{3} \mathrm{O}_{4}$ MPs were prepared using a method previously described with some modifications (10). The monodispersed $\mathrm{Fe}_{3} \mathrm{O}_{4}$ MPs were dispersed in a non-polar organic solvent and synthesized using a high-temperature organic solution phase reaction. Iron (III) acetylacetonate (Fe (acac)3, 2 mmol; 99.9\%), 1,2-hexadecanediol (10 mmol; $90 \%)$, oleic acid (6 mmol; $99 \%)$, oleylamine (6 mmol; 70\%) and 1-octadecene (20 ml; 90\%) (all from Sigma-Aldrich) were mixed and magnetically stirred under a nitrogen atmosphere. The mixture was heated to $200^{\circ} \mathrm{C}$ for $2 \mathrm{~h}$ and then heated to reflux $\left(\sim 300^{\circ} \mathrm{C}\right)$ for an additional hour. The black-colored mixture was cooled down to room temperature. Ethanol (40 $\mathrm{ml})$ was added to the mixture under ambient conditions, and a black material was precipitated and separated via centrifugation $(12,000 \mathrm{rpm}, 30 \mathrm{~min})$. The black product was redispersed in hexane in the presence of oleic acid $(\sim 0.05 \mathrm{ml})$ and oleylamine $(\sim 0.05 \mathrm{ml})$. Centrifugation $(6,000 \mathrm{rpm}, 10 \mathrm{~min})$ was applied to remove any undispersed residue. The product was then precipitated with ethanol, centrifuged $(10,000 \mathrm{rpm}$, $20 \mathrm{~min}$ ) to remove the solvent and redispersed in organic solvents, such as n-hexane and chloroform. The resulting $\mathrm{Fe}_{3} \mathrm{O}_{4}$ MPs dispersed in chloroform were encapsulated with a PEG-phospholipid shell to make them biocompatible. Typically, $2 \mathrm{ml}$ of the organic dispersible $12 \mathrm{~nm}$ sized $\mathrm{Fe}_{3} \mathrm{O}_{4}$ MPs in chloroform $(5 \mathrm{mg} / \mathrm{ml})$ was mixed with $1 \mathrm{ml}$ of chloroform solution containing $10 \mathrm{mg}$ 1,2-distearoylsn-glycero-3-phosphoethanolamine-N-[methoxy(PEG)-2000] (mPEG-2000 PE) (Avanti Polar Lipids, Inc.) at a ratio of 5:1. After complete evaporation of the chloroform, the residue was incubated at $80^{\circ} \mathrm{C}$ in a vacuum for $1 \mathrm{~h}$. Five milliliters of water were added, which produced a clear and dark-brown suspension containing PEG-PE micelles. As this suspension contained both empty micelles and micelles containing MPs, the empty micelles were removed by ultracentrifugation. The micelles containing MPs formed a pellet, whereas the empty micelles remained suspended. The supernatant was discarded, and the MP micelles were resuspended in phosphate-buffered saline (PBS). In the present study, $50 \mu \mathrm{g} / \mathrm{ml}$ of $\mathrm{Fe}_{3} \mathrm{O}_{4} \mathrm{MPs}$ were added to the osteogenic differentiation medium.

Immunohistochemical staining. The cells cultured on the cover slide were fixed for $20 \mathrm{~min}$ at $4^{\circ} \mathrm{C}$ using $10 \%$ neutral-buffered formalin and subsequently washed 3 times with PBS ( $\mathrm{pH}$ 7.2). These cover slides were then incubated with anti-osteocalcin (predilution, AM 386; BioGenex, San Ramon, CA, USA), anti-osteopontin (1:1,000 dilution) and anti-osteonectin (1:500 dilution; AB 1858, Chemicon, Carlsbad, CA, USA) antibodies for $24 \mathrm{~h}$, followed by development using EnVision Plus reagent (Dako, Carpinteria, CA, USA), diaminobenzidine as a chromogen and Mayer's hematoxylin as a counterstain.

von Kossa staining. The mineralized matrix of the cells was assessed using 5\% silver nitrate (Sigma-Aldrich) under ultraviolet light for $60 \mathrm{~min}$, followed by the addition of 3\% sodium thiosulphate (Sigma-Aldrich) for $5 \mathrm{~min}$ and then counterstaining with Van Gieson (Sigma-Aldrich) for $5 \mathrm{~min}$. With this staining method, the mineral matrix is stained black and the osteoid (unmineralized matrix) is stained red. 


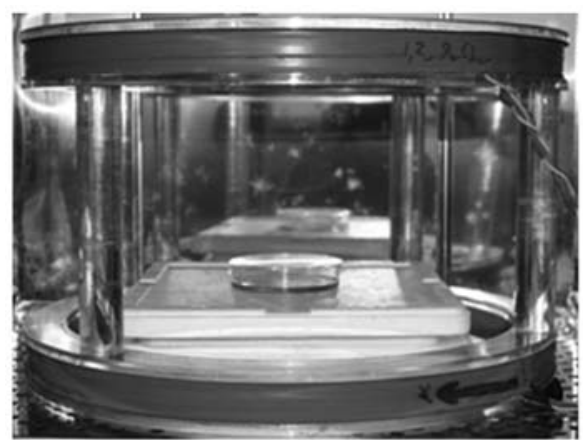

Coil

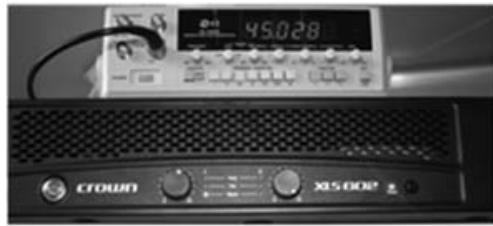

Generator

Figure 1. Schematic representation of the sinusoidal electromagnetic field device, which was placed in a $5 \% \mathrm{CO}_{2}$ and a $37^{\circ} \mathrm{C}$ incubator.

Table I. Primers used for RT-qPCR.

\begin{tabular}{lll}
\hline Genes & \multicolumn{1}{c}{ Upstream primer sequence } & Downstream primer sequence \\
\hline GAPDH & 5'-ACC ACA GTC CAT GCC ATC AC-3' & 5'-TCC ACC ACC CTG TTG CTG TA-3' \\
Collagen I & 5'-GAA AAC ATC CCA GCC AAG AA-3' & 5'-CAG GTT GCC AGT CTC CTC AT-3' \\
Collagen III & 5'-CAG GTG AAC GTG GAG CTG C-3' & 5'-TGC CAC ACG TGT TTC CGT GG-3' \\
Osteonectin & 5'-CCA GAA CCA CCA CTG CAA AC-3' & 5'-GGC AGG AAG AGT CGA AGG TC-3' \\
Osteocalcin & 5'-AGG GGA AGA GGA AAG AAG GG-3' & 5'-CCA GGC GCT ACC TGT ATC AA-3' \\
Osteopontin & 5'-TCG CAG ACC TGA CAT CCA GT-3' & 5'-TCG GAA TGC TCA TTG CTC TC-3' \\
BMP-2 & 5'-GTC CAG CTG TAA GAG ACA CC-3' & 5'-GTA CTA GCG ACA CCC ACAAC-3' \\
Runx-2 & 5'-CTC ACT ACC ACA CCT ACC TG-3' & 5'-TCA ATA TGG TCG CCAAAC AGA TTC-3' \\
BSP & 5'-CAC AGC CTC ATC TTC ATG G-3' & 5'-GCA TCT CAT AGT GCA TCT GG-3' \\
CACNA1C & 5'-ACA GTG ACC AGT GTG GTG GA-3' & 5'-CGT AGC CTC TGG AGA ACC TG-3' \\
CACNA1E & 5'-GTT CGG CCG CGA TCA CCT TTG T-3' & 5'-GGC GGC CAA TCG ATG AGC TTC T-3' \\
CACNA1G & 5'-CGG CAA CTAC GTG CTC TTC A-3' & 5'-GTG ACT TCA TCT CGT GGG CC-3' \\
CACNA1I & 5'-CGT TGT CAT AGC GAC CCA GTT-3' & 5'-CAC AGC TCT CTT CCC CGA GTG A-3'
\end{tabular}

BMP-2, bone morphogenic protein-2; Runx-2, runt-related transcription factor-2; BSP, bone sialoprotein; CACNA1C, alpha 1C subunit of the L-type voltage-gated calcium channel; CACNA1E, alpha 1E subunit of the R type voltage-dependent calcium channel; CACNA1G, alpha 1G subunit of the T-type voltage-dependent calcium channel; CACNA1I, alpha 1I subunit of T-type voltage-dependent calcium channel.

$R T$ - $q P C R$. Total RNA was isolated from the cells using $500 \mu 1$ TRIzol reagent (Sigma-Aldrich). Subsequently, $100 \mu \mathrm{l}$ of chloroform were added and the solution was mixed and incubated for $3 \mathrm{~min}$. Following centrifugation $\left(12,000 \mathrm{rpm}, 4^{\circ} \mathrm{C}\right.$ for $15 \mathrm{~min}$ ), the upper phase was transferred to a new tube and $500 \mu \mathrm{l}$ of isopropanol were added. After an incubation period of $10 \mathrm{~min}$ and another centrifugation step $\left(14,000 \mathrm{rpm}, 4^{\circ} \mathrm{C}\right.$ for $10 \mathrm{~min})$, the supernatant was discarded. The pellet was washed with $1 \mathrm{ml}$ of $70 \%$ ethanol and centrifuged $\left(9,500 \mathrm{rpm}, 4^{\circ} \mathrm{C}\right.$ for $5 \mathrm{~min})$. The supernatant was discarded and the pellet was dried. Following the addition of $20 \mu \mathrm{l}$ of diethylpyrocarbonate (DEPC)-treated water, the pellet was dissolved for $10 \mathrm{~min}$ on ice. The amount and purity of total RNA were determined using a nanodrop spectrophotometer (Hankyong National University). Reverse transcriptase (RT) reactions were used to synthesize the cDNA from $1 \mu \mathrm{g}$ of total RNA using an Advantage RT-for-RCR kit (Clontech Laboratories, Inc., Palo Alto, CA, USA). RT-PCR was routinely performed. The sample was cooled to $-20^{\circ} \mathrm{C}$ and stored until further analysis. For PCR, the primers were purchased from Bioneer Corp. (Deajeon, Korea). The primer sequences used for quantitative PCR are listed in Table I.

Western blot analysis. After 10 days of cell culture, the cells were lysed with RIPA buffer containing $50 \mathrm{mM}$ Tris- $\mathrm{HCl}$, $\mathrm{pH}$ 8.0, $150 \mathrm{mM} \mathrm{NaCl}, 1 \% \mathrm{NP}-40,0.5 \%$ sodium deoxycholate, $0.1 \%$ SDS (Sigma-Aldrich), protease inhibitors (Complete ${ }^{\mathrm{TM}}$; Roche Diagnostics, Mannheim, Germany). Protein $(30 \mu \mathrm{g})$ was then separated by SDS-polyacrylamide gel electrophoresis and blotted onto nitrocellulose membranes, which were then blocked with $5 \%$ skim milk in phosphate-buffered saline (PBS) containing $0.2 \%$ Tween-20. Western blot analysis was performed according to the manufacturer's instructions (Abcam, Cambridge, MA, USA) with the following primary antibodies: anti-bone sialoprotein (BSP, ab52128; Abcam), anti-bone morphogenetic protein 2 (BMP-2, ab17885; Abcam), 
anti-osteopontin (ab63856; Abcam), anti-osteonectin (ab14174; Abcam), anti-phosphorylated extracellular signal-regulated kinase (p-ERK, \#9101; Cell Signaling Technology, Danvers, MA, USA) and anti-actin (ab3280; Abcam). The blots were incubated with the primary antibodies at a dilution of 1:5,000 and then further incubated with horseradish peroxidase-conjugated secondary antibody (\#7076; Cell Signaling Technology).

Cell surface antigen analysis by fuorescence-activated cell sorting (FACS). Antibodies against the human antigens, CD73 and CD90, were purchased from BD Biosciences (San Jose, CA, USA) and the antibody against CD105 was purchased from Ancell Corp. (Bayport, MN, USA). A total of $5 \times 10^{5}$ cells were resuspended in $200 \mu 1$ of PBS and incubated with fluorescein isothiocyanate (FITC)- or phycoerythrin (PE)-conjugated antibodies for $20 \mathrm{~min}$ at room temperature (or for $45 \mathrm{~min}$ at $4^{\circ} \mathrm{C}$ ). The fluorescence intensity of the cells was evaluated using a flow cytometer (FACScan; BD Biosciences), and the data were analyzed using CellQuest software (BD Biosciences).

Proliferation and activity assay of hBM-MSCs. Cell proliferation and activity were measured using a cell counter (Scepter ${ }^{\mathrm{TM}}$; Millipore Corp., Billerica, MA, USA) and a 3-(4,5-dimethylthiazol-2-yl)-2,5-diphenyl tetrazolium bromide (MTT; Sigma) assay. For the MTT assay, the cells were cultured in a 6-well plate, and each well was supplemented with MTT $(3 \mathrm{mg} / \mathrm{ml})$ $(\mathrm{n}=4)$. The plates were then incubated in the dark at $37^{\circ} \mathrm{C}$ in an atmosphere containing $5 \% \mathrm{CO}_{2}$ for $2 \mathrm{~h}$ and the supernatant was aspirated. Dimethylsulfoxide (DMSO) was added and the 6-well plate was shaken slowly for $5 \mathrm{~min}$. The absorption was measured at $570 \mathrm{~nm}$.

LDH assay. LDH activity was measured using an LDH-LQ kit (Asan Pharmaceutical Inc., Seoul, Korea). Briefly, after 7 days of culture, $20-\mu 1$ aliquots of medium and $50-\mu 1$ of working solution were mixed and incubated in the dark at room temperature for $30 \mathrm{~min}$. The reaction was terminated by the addition of stop solution $(1 \mathrm{~N} \mathrm{HCl})$ and the absorbance was measured at $570 \mathrm{~nm}$.

ALP assay. ALP deposition was measured using a SensoLyte ${ }^{\circledR}$ $p$ NPP Alkaline Phosphatase Assay kit (AnaSpec Inc., Fremont, CA, USA). Following sample preparation, the samples were mixed with $p$ NPP substrate solution. The mixtures were incubated for 30-60 min and stop solution was added. The amount of ALP was quantified by ELISA at $405 \mathrm{~nm}$.

\section{Results and Discussion}

Morphology and characterization of hBM-MSCs. The hBMMSCs were cultured in osteogenic differentiation medium and were treated with MPs or exposed to EMFs over a period of 7 days. The morphology of the hBM-MSCs during osteogenesis under the different experimental conditions [control (no treatment), MP incorporation, EMF exposure, MP incorporation and exposure to EMF] is shown in Fig. 2. No morphological changes or necrosis of the hBM-MSCs were observed following the induction of osteogenic differentiation (Fig. 2). Therefore, MP incorporation, EMF exposure and MP incorporation in conjunction with exposure to EMF did not induce any cytotoxic effects.

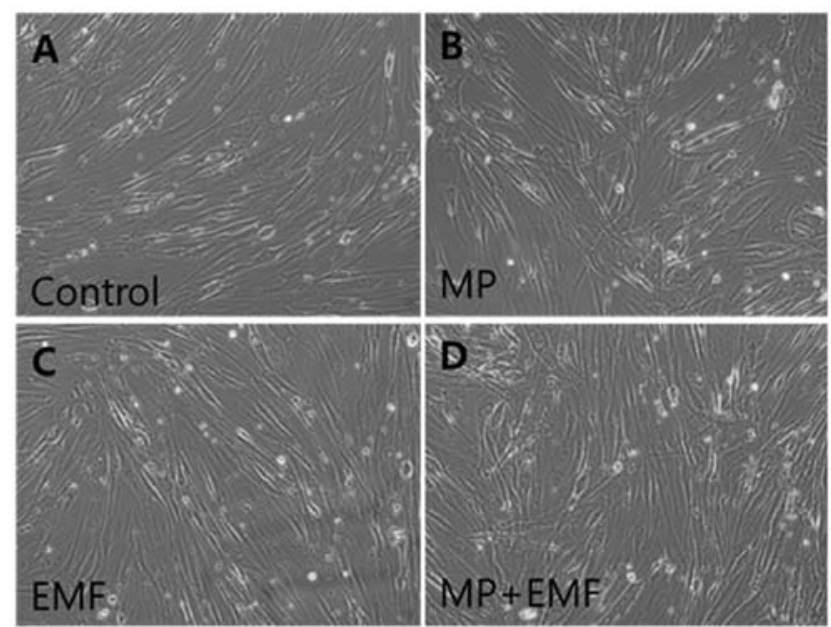

Figure 2. Morphology of the hBM-MSCs following the induction of osteogenic differentiation. Cells cultured in (A) osteogenic differentiation medium, with (B) magnetic nanoparticle (MP) incorporatiohn, (C) exposure to electronic magnetic fields (EMFs) and (D) MP incorporation and exposure to EMFs $(\mathrm{MP}+\mathrm{EMF})$ for 7 days. Original magnification, $\mathrm{x} 100$.

Table II. Staining results of the osteogenic markers.

\begin{tabular}{lcccc}
\hline & Control & MP & EMF & MP + EMF \\
\hline Osteocalcin & - & + & ++ & ++ \\
Osteonectin & + & ++ & +++ & +++ \\
Osteopontin & + & +++ & ++ & +++ \\
von Kossa & - & ++ & + & ++ \\
\hline
\end{tabular}

-, no or weak staining; + , low intensity; ++ , moderate intensity; and +++ , strong intensity.

Immunohistochemical staining. To evaluate the protein expression levels of osteogenic markers and mineralization, immunohistochemical staining was performed. Osteocalcin protein is specifically synthesized by osteoblasts and is a marker of osteoblast differentiation during the later stages of bone formation (11). Osteonectin is a glycoprotein in the bones which binds sodium. It is secreted by osteoblasts during bone formation, initiating mineralization and promoting mineral crystal formation (12). Osteopontin is a highly phosphorylated sialoprotein, which is a prominent component of the mineralized extracellular matrices of bones (13). The osteogenic markers (osteocalcin, osteopontin and osteonectin) were strongly expressed in the cells treated with MPs, in those exposed to EMFs and in the cells treated with MPs and exposed to EMFs compared to the control group (Fig. 3A-L). The results from immunohistochemical staining of the expression levels of the osteogenic markers are presented in Table II.

von Kossa staining is widely used to quantify mineralization (14-16). It is generally believed that, in von Kossa's technique, silver cations react with phosphates and carbonates in calcium deposits (17). We used von Kossa staining for the investigation of the mineralization of hBM-MSCs during osteogenesis (Fig. 3M-P). The untreated hBM-MSCs (control) or those exposed to EMFs growing in osteogenic medium exhibited a small amount of matrix mineralization; however, 


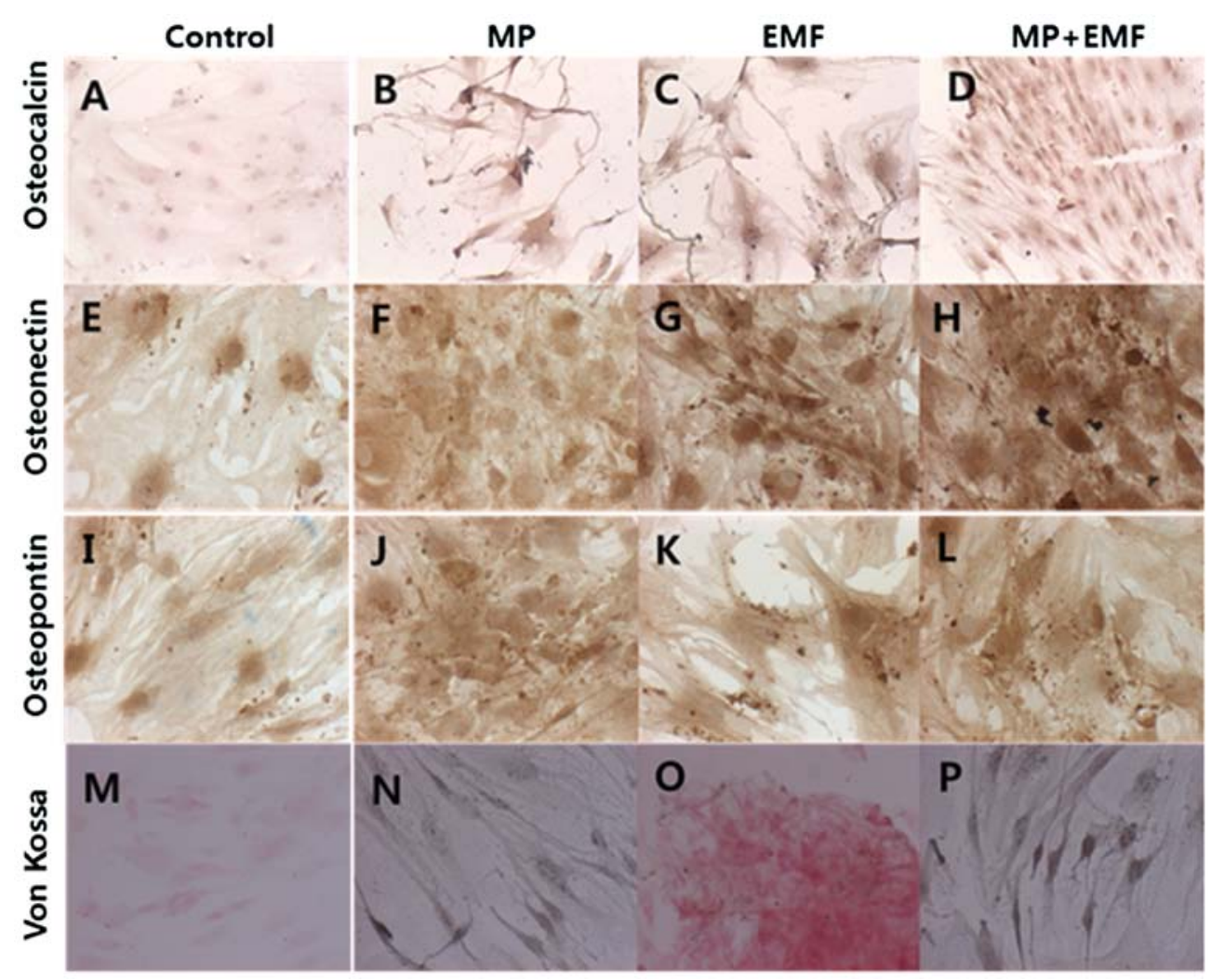

Figure 3. Immunocytochemical and von Kossa staining of the BM-MSCs following the induction of osteogenic differentiation for 7 days. (A-D) Ostoecalcin; (E-H) osteonectin; (I-L) osteopontin; and (M-P) von Kossa staining.

the hBM-MSCs treated with MPs and those treated with MPs and exposed to EMFs exhibited a stronger matrix mineralization compared to the untreated controls on day 7. The amount of mineralization in each treatment group is shown in Table II.

$R T-q P C R$. The mRNA expression levels of osteogenesisrelated genes from the hBM-MSCs following the induction of osteogenic differentiation for 3 days is shown in Fig. 4A. The osteoblast markers, osteocalcin, osteopontin and osteonectin were highly expressed in the hBM-MSCs treated with MPs and exposed to EMFs. We also examined the expression levels of major bone matrix protein genes, such as collagen I, collagen III, BMP-2 and BSP. BMP-2 induces osteoblastic differentiation by acting directly on MSCs and is clinically used to induce bone formation although high doses are required (18). BSP is a highly post-translationally modified acidic phosphorus protein normally expressed in mineralized tissue, such as bone and dentin (19). The representative osteogenesis-related genes were highly expressed in the hBM-MSCs treated with MPs, those exposed to EMFs and in those treated with MPs and exposed to EMFs. The results of our experiments indicated that the expression levels of BSP, OPN, OCN, and collagen I were significantly increased in the EMF with MP exposure group. Osteogenic gene expression can be used as an early index during osteogenesis. BSP is a late stage marker of osteoblast differentiation and an early stage marker of matrix mineralization. OPN is expressed throughout matrix maturation, followed first by BSP and finally by OCN, which characterizes the post-proliferate phase. The OCN protein is specifically synthesized by osteoblasts and is a marker of osteoblast differentiation during the later stages of bone formation. Collagen I is the most abundant protein in the bone matrix and is an early marker of osteoblastic differentiation and the major organic component of the mineralized bone matrix. Additionally, the mRNA expression of the transcription factor Runx-2 was measured by RT-qPCR. Runx-2 is involved in the production of bone matrix proteins as it is able to upregulate the expression of major bone matrix protein genes leading to an increase in immature osteoblasts from pluripotent stem cells; the immature osteoblasts form immature bone (20-25). Runx-2 was strongly expressed in the hBM-MSCs treated with MPs, those exposed to EMFs and in those treated with MPs and exposed to EMFs compared to the untreated control.

Recent studies have suggested that calcium activation affects bone formation. Klar et al (26) reported that the spontaneous induction of bone formation is initiated by a local peak of calcium-activating stem cell differentiation and the induction of bone formation. Moreover, Wen et al (27) mentioned that the inhibition of the L-type voltage-dependent calcium channel (VDCCL) downregulates the proliferation and osteogenic differentiation of rat MSCs (rMSCs), but promotes apoptosis. These results suggest that VDCCL plays a crucial role in the proliferation and osteogenic differentiation of rMSCs. Based on this knowledge, we investigated the effects of MPs and EMF exposure on the expression of calcium channel-related genes in the hBM MSCs during osteogenesis. After 3 days of osteogenesis and treatment with MPs and EMF exposure, the mRNA expression levels of CACNA1C and CACNA1I in the cells treated with MPs, those exposed to EMFs and in those treated with MPs and exposed to EMFs were slightly higher compared to the control group (Fig. 4B). In addition, the expression levels 
A
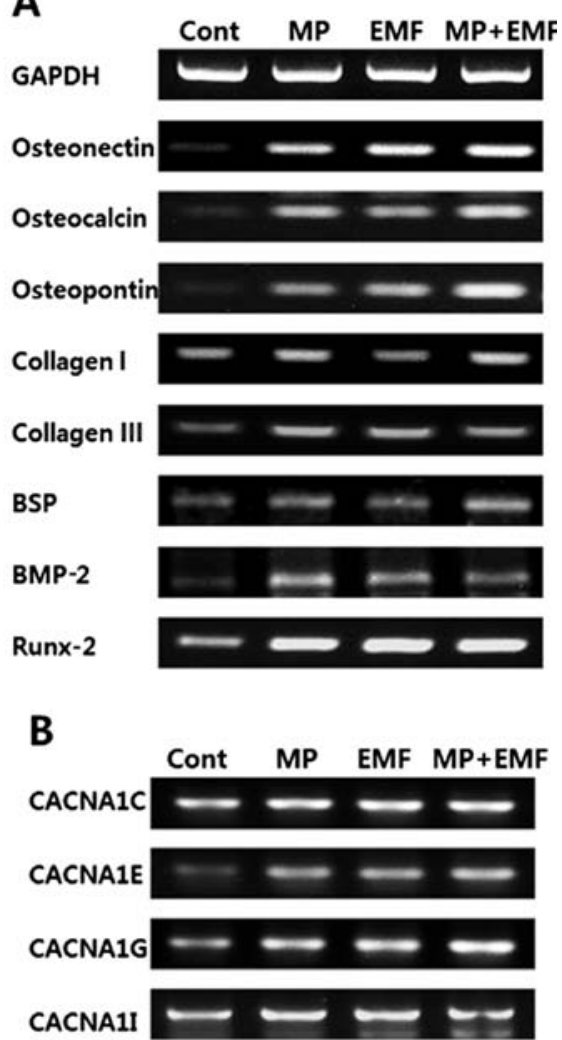

Figure 4. Determinatino of gene expression levels by RT-qPCR in the hBM-MSCs following the induction of osteogenic differentiation for 3 days. Electrophoretic RT-qPCR analysis of (A) osteogenesis-related genes and (B) calcium channel-related genes.

of CACNA1E and CACNA1G were significantly higher in the cells treated with MPs and exposed to EMFs (Fig. 4B). This suggested that the hBM-MSC calcium channel was activated during osteogenic differentiation.

Western blot analysis. To examine osteogenic differentiation, osteogenesis-related proteins were detected by western blot analysis at day 7 of induction. As shown in Fig. 5, the expression levels of osteogenesis-related proteins (BSP, BMP-2, osteopontin and osteonectin) were increased in the cells treated with MPs, those exposed to EMFs and in those treated with MPs and exposed to EMFs compared to the control group.

To examine the potential p-ERK activation during osteogenesis, western blot analysis was performed. Several studies have reported that $\mathrm{p}$-ERK activation is an essential mediator of growth factor-induced cell proliferation and differentiation in various cell types, including osteoblasts (28-31). Kapur et al (32) demonstrated that the activation of ERK1/2 by mechanical stimuli is involved in collagen synthesis and osteopontin production. In the present study, after 7 days of osteogenesis with MP incorporation and EMF exposure, the expression of p-ERK was increased and it was highly expressed in the cells treated with MPs and exposed to EMFs (Fig. 5).

FACS analysis. Several antigens are known as MSC surface markers. The antibodies against CD73 (membrane-bound ecto-5'-nucleotisidase), CD90 (Thy-1), CD105 (endoglin) have been reported to react with undifferentiated MSCs (33-35).

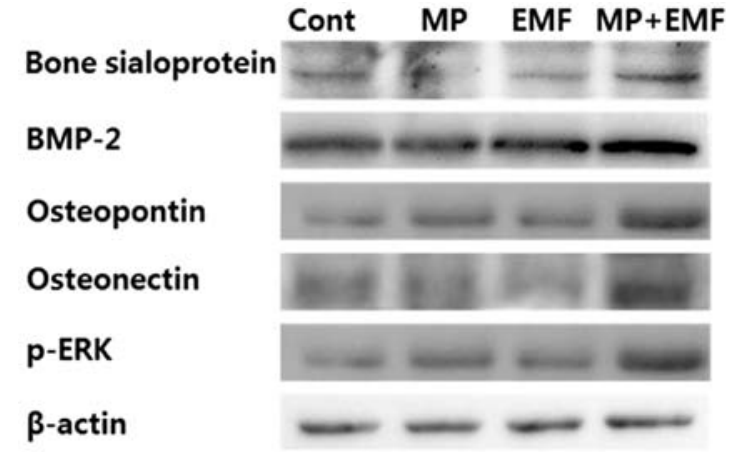

Figure 5. Western blot analysis of hBM-MSCs following the induction of osteogenic differentiation for 7 days. Whole cell lysates were immunoblotted with antibody of osteogenesis-related proteins (bone sialoprotein, BMP-2, osteopontin, osteonectin), p-ERK and $\beta$-actin.

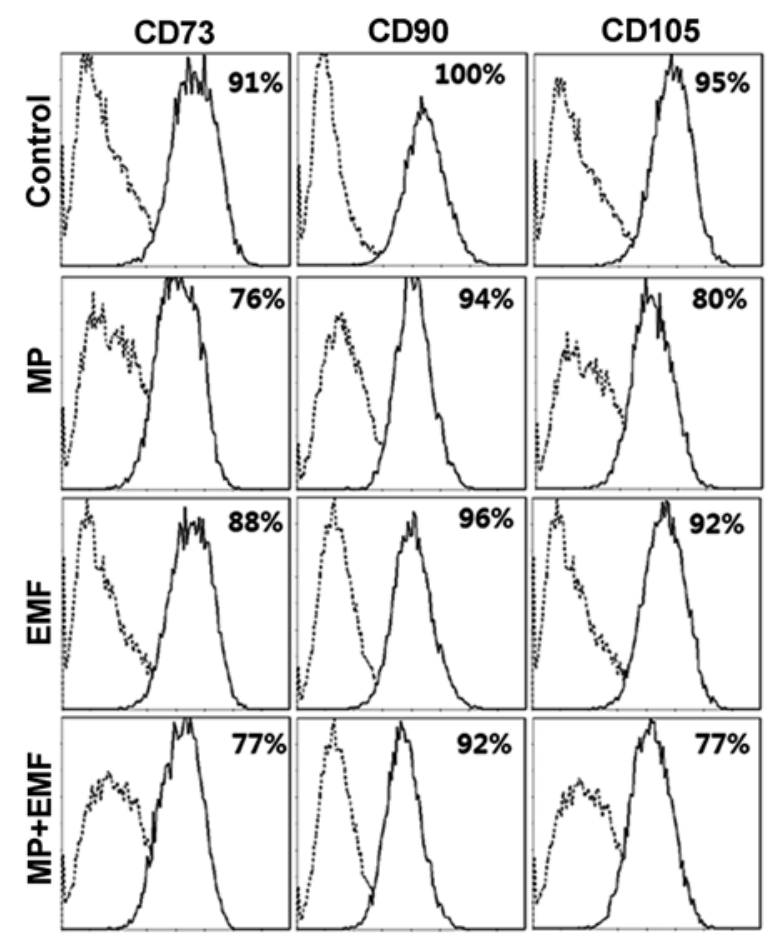

Figure 6. Fluorescence-activated cell sorting (FACS) analysis of hBM-MSC surface markers following the induction of osteogenic differentiation for 7 days.

To investigate whether MP incorporation and exposure to EMFs affects hBM-MSC differentiation, FACS analysis was performed for the hBM-MSC markers, CD73, CD90 and CD105 (Fig. 6). The expression level of CD73 was $91 \%$ in the control group, $76 \%$ in the MP incorporation group, $88 \%$ in the EMF-exposed group and $77 \%$ in the MP incorporation with exposure to EMF group after 7 days. The expression level of CD90 was $100 \%$ in the control group, $94 \%$ in the MP incorporation group, $96 \%$ in the EMF-exposed group and $77 \%$ in the MP incorporation with exposure to EMF group. The expression of CD105 was $95 \%$ in the control group, $80 \%$ in the MP incorporation group, $92 \%$ in the EMF-exposed group and $77 \%$ in the MP incorporation with exposure to EMF group. The expression levels of hBM-MSC surface antigens were decreased in the cells treated with MPs, those exposed to EMFs 

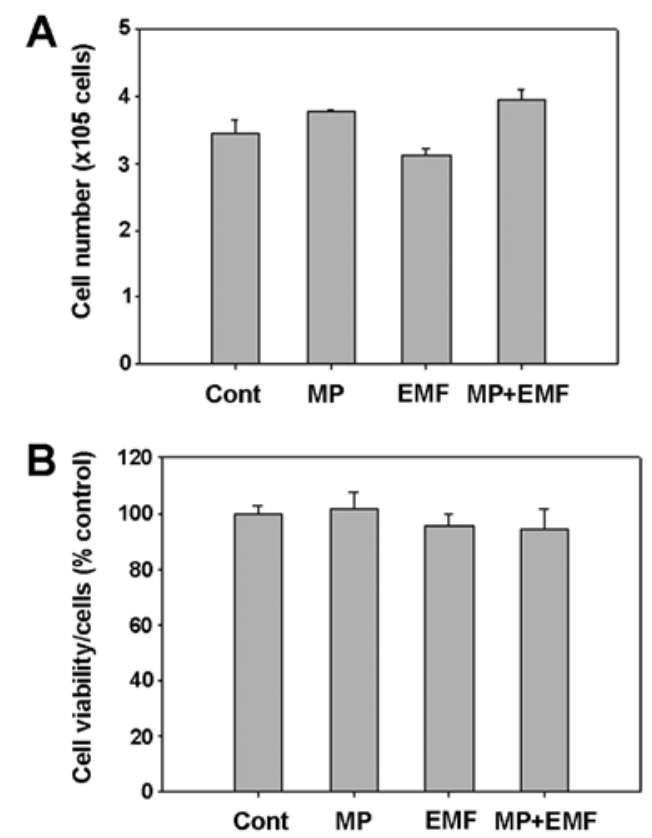

Figure 7. Effect of magnetic nanoparticle (MPs) and electronic magnetic fields (EMFs) on the proliferation of hBM-MSCs during osteogenic differentiation. (A) hBM-MSCs were cultured for 7 days, and their proliferation was measured by counting cell numbers. (B) MTT assay was performed for the assessment of the viability of hBM-MSCs under the different culture conditions (control, MP, EMF and MP + EMF) after 7 days.
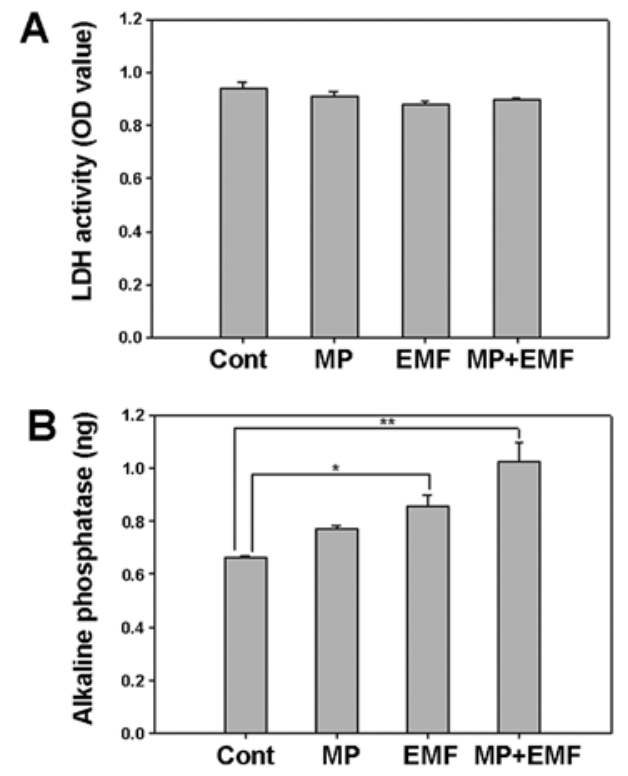

Figure 8. Effect of different culture conditions on cytotoxicity and alkaline phosphatase (ALP) activity of hBM-MSCs during osteogenic differentiation for 7 days. (A) Cytotoxicity was measured by a lactate dehydrogenase (LDH) assay kit. (B) ALP assay was performed for the determination of ALP activity. $\left({ }^{*} \mathrm{P}<0.05,{ }^{* *} \mathrm{P}<0.05\right)$.

and in those treated with MPs and exposed to EMFs compared to the control group. In conclusion, our data indicate that MP incorporation and EMF exposure alter MSC surface antigen expression, suggesting that the hBM-MSCs differentiate into a specific cell type, thus changing their cell fate.

Cell proliferation and activity assay. The cell number was determined to be approximately $3.4 \times 10^{5}$ cells in the control group, $3.7 \times 10^{5}$ cells in the MP incorporation group, $3.1 \times 10^{5}$ cells

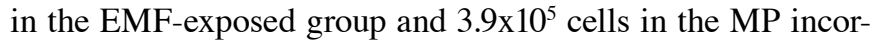
poration with exposure to EMF group after 7 days (Fig. 7A).

As shown by the cell counting results, the cells treated with MPs and those treated with MPs and exposed to EMFs exhibited slightly accelerated cell growth compared to the control group, while the group exposed to EMFs showed a more decelerated cell growth compared to the control group (Fig. 7A).

In addition, cell viability and toxicity to hBM-MSCs were measured by MTT assay 7 days after osteogenesis. MTT assay provides valuable information as to the potential cell viability and cytotoxic effects. Although the cell numbers were decreased following exposure to EMFs and increased following treatment with MPs and treatment with MPs in conjunction with EMF exposure, the cell mitochondrial activity of the 4 experimental groups was similar (Fig. 7B). Morevoer, these results demonstrated that no cytotoxic effects were detected in the hBM-MSCs treated with MPs and in those exposed to EMFs during osteogenesis.

LDH and ALP activity. LDH is a cytoplasmic catalytic enzyme related to the reversible conversion between pyruvic and lactic acid. LDH is released through the cell membrane when it is damaged (36). Therefore, less LDH release means less cellular damage. Thus, the media were collected and analyzed after 7 days in order to examine cellular damage.

In the cells treated with MPs and in those treated with MPs and exposed to EMFs there was a decrease in LDH secretion (Fig. 8A). The LDH activity of the 4 experimental groups was similar, which suggests that treatment with MPs and exposrue to EMFs did not affect LDH secretion and did not induce damage to the cell membrane.

ALP is an early mineralization-related protein marker for the osteogenesis of osteoblasts (37). The effects of MP incorporation and exposure to EMFs on cellular ALP activity during osteogenesis were examined by measuring the ALP reaction products in the untreated cells (control), the cells treated with MPs, those exposed to EMFs, and in those treated with MPs and exposed to EMFs over time. Several ALP-positive cells were observed in the cells exposed to EMFs and in those treated with MPs and exposed to EMFs (Fig. 8B). These results suggest that MP incorporation in conjunction with exposure to EMFs increases ALP activity in hBM-MSCs during osteogenesis.

In conclusion, in the present study, the effects of treatment with MPs in conjunction with exposure to EMFs on cell differentiation were investigated. We treated hBM-MSCs with $50 \mu \mathrm{g} / \mathrm{ml}$ of $\mathrm{Fe}_{3} \mathrm{O}_{4}$ MPs or exposed them to a frequency of $45 \mathrm{~Hz}$ and an intensity of $1 \mathrm{mT}$ EMF twice every $8 \mathrm{~h}$ per day for 7 days. No morphological changes and no cytotoxic effects were observed during osteogenesis. The expression of osteogenic markers was detected by immunohistochemical staining, RT-qPCR and western blot analysis, demonstrating an increase in expression. FACS analysis indicated that treatment with MPs and EMF exposure reduced the expression of MSC surface markers; this suggests the possibility of hBM-MSC differentiation. The mineralization of hBM-MSCs in the MP incorporation group or EMF-exposed group was observed by ALP assay. Taken together, these results suggest that the treatment of hBM-MSCs with MP or their exposure to EMFs increases osteogenic differentiation, and that MP 
incorporation in conjunction with EMF exposure is more effective in enhancing osteogenic differentiation. The results of our study demonstrate that MPs may be potentially used for medical instruments or scaffold materials, and that EMFs can be used for the rehabilitation of osteogenic wounds.

\section{Acknowledgements}

The present study was supported by the Pioneer Research Center Program through the National Research Foundation of Korea funded by the Ministry of Science, ICT and Future Planning, Republic of Korea (grant number 2009-0082941).

\section{References}

1. Sadan O, Melamed E and Offen D: Bone-marrow-derived mesenchymal stem cell therapy for neurodegenerative diseases. Expert Opin Biol Ther 9: 1487-1497, 2009.

2. Jiang Y, Jahagirdar BN, Reinhardt RL, Schwartz RE, Keene CD Ortiz-Gonzalez XR, Reyes M, Lenvik T, Lund T, Blackstad M, Du J, Aldrich S, Lisberg A, Low WC, Largaespada DA and Verfaillie C: Pluripotency of mesenchymal stem cells derived from adult marrow. Nature 418: 41-49, 2002.

3. Bielby R, Jones E and McGonagle D: The role of mesenchymal stem cells in maintenance and repair of bone. Injury 38 (Suppl 1): S26-S32, 2007.

4. Fukada E and Yasuda I: On the piezoelectric effect of bone. J Phys Soc Japan 12: 1158-1162, 1957.

5. Bassett CA and Pawluk RJ: Effects of electric currents on bone in vivo. Nature 204: 652-654, 1964.

6. Tsai MT, Li WJ, Tuan RS and Chang WH: Modulation of osteogenesis in human mesenchymal stem cells by specific pulsed electromagnetic field stimulation. J Orthop Res 27: 1169-1174, 2009.

7. Sun LY, Hsieh DK, Lin PC, Chiu HT and Chiou TW: Pulsed electromagnetic fields accelerate proliferation and osteogenic gene expression in human bone marrow mesenchymal stem cells during osteogenic differentiation. Bioelectromagnetics 31: 209-219, 2010.

8. Pankhurst QA, Connolly J, Jones S and Dobson J: Applications of magnetic nanoparticles in biomedicine. J Phys D Appl Phys 36: R167-R181, 2003.

9. Cartmell SH, Dobson J, Verschueren SB and El Haj AJ Development of magnetic particle techniques for long-term culture of bone cells with intermittent mechanical activation. IEEE Trans Nanobioscience 1: 92-97, 2002.

10. Cho H, Choi YK, Lee DH, Park HJ, Seo YK, Jung H, Kim SC Kim SM and Park JK: Effects of magnetic nanoparticle-incorporated human bone marrow-derived mesenchymal stem cells exposed to pulsed electromagnetic fields on injured rat spinal cord. Biotechnol Appl Biochem 60: 596-602, 2013.

11. Ducy P, Desbois C, Boyce B, Pinero G, Story B, Dunstan C, Smith E, Bonadio J, Goldstein S, Gundberg C, Bradley A and Karsenty G: Increased bone formation in osteocalcin-deficient mice. Nature 382: 448-452, 1996.

12. Kelm RJ Jr, Hair GA, Mann KG and Grant BW: Characterization of human osteoblast and megakaryocyte-derived osteonectin (SPARC). Blood 80: 3112-3119, 1992.

13. Sodek J, Ganss B and McKee M: Osteopontin. Crit Rev Oral Biol Med 11: 279-303, 2000

14. von Kossa J: Über die im Organismus künstlich erzeugbaren Verkalkungen. Beit Path Anat 29: 163-202, 1901 (In German).

15. Bills C, Eisenberg H and Pallante SL: Complexes of organic acids with calcium phosphate: the von Kossa stain as a clue to the composition of bone mineral. Johns Hopkins Med J 128: 194-207, 1971.

16. Puchtler H and Meloan S: On the chemistry of formaldehyde fixation and its effects on immunohistochemical reactions. Histochemistry 82: 201-204, 1985.

17. Meloan SN and Puchtler H: Chemical mechanisms of staining methods: von Kossa's technique: what von Kossa really wrote and a modified reaction for selective demonstration of inorganic phosphates. J Histotechnol 8: 11-13, 1985.
18. Schwartz Z, Simon B, Duran M, Barabino G, Chaudhri R and Boyan B: Pulsed electromagnetic fields enhance BMP-2 dependent osteoblastic differentiation of human mesenchymal stem cells. J Orthop Res 26: 1250-1255, 2008.

19. Schwartz Z, Lohmann C, Oefinger J, Bonewald L, Dean D and Boyan B: Implant surface characteristics modulate differentiation behavior of cells in the osteoblastic lineage. Adv Dent Res 13: 38-48, 1999.

20. Ogawa E,Inuzuka M,Maruyama M, Satake M,Naito-Fujimoto M, Ito $\mathrm{Y}$ and Shigesada $\mathrm{K}$ : Molecular cloning and characterization of PEBP $2 \beta$, the heterodimeric partner of a novel Drosophila runt-related DNA binding protein PEBP2 $\alpha$. Virology 194: 314-331, 1993.

21. Miyoshi H, Shimizu K, Kozu T, Maseki N, Kaneko Y and Ohki M: $t(8 ; 21)$ breakpoints on chromosome 21 in acute myeloid leukemia are clustered within a limited region of a single gene, AML1. Proc Natl Acad Sci USA 88: 10431-10434, 1991.

22. Komori T, Yagi H, Nomura S, Yamaguchi A, Sasaki K, Deguchi K, Shimizu Y, Bronson R, Gao YH, Inada M, Sato M, Okamoto R, Kitamura Y, Yoshiki S and Kishimoto T: Targeted disruption of $C b f a l$ results in a complete lack of bone formation owing to maturational arrest of osteoblasts. Cell 89: 755-764, 1997.

23. Ducy P, Zhang R, Geoffroy V, Ridall AL and Karsenty G: Osf 2/Cbfa1: a transcriptional activator of osteoblast differentiation. Cell 89: 747-754, 1997.

24. Otto F, Thornell AP, Crompton T, Denzel A, Gilmour KC, Rosewell IR, Stamp GW, Beddington RS, Mundlos S, Olsen BR, Selby PB and Owen MJ: Cbfal, a candidate gene for cleidocranial dysplasia syndrome, is essential for osteoblast differentiation and bone development. Cell 89: 765-771, 1997.

25. Komori T: Regulation of bone development and extracellular matrix protein genes by RUNX2. Cell Tissue Res 339: 189-195, 2010.

26. Klar RM, Duarte R, Dix-Peek T, Dickens C, Ferretti C and Ripamonti U: Calcium ions and osteoclastogenesis initiate the induction of bone formation by coral-derived macroporous constructs. J Cell Mol Med 17: 1444-1457, 2013.

27. Wen L, Wang Y, Wang H, Kong L, Zhang L, Chen X and Ding Y: L-type calcium channels play a crucial role in the proliferation and osteogenic differentiation of bone marrow mesenchymal stem cells. Biochem Biophys Res Commun 424: 439-445, 2012.

28. Lai CF, Chaudhary L, Fausto A, Halstead LR, Ory DS, Avioli LV and Cheng SL: Erk is essential for growth, differentiation, integrin expression, and cell function in human osteoblastic cells. J Biol Chem 276: 14443-14450, 2001.

29. Azuma N, Duzgun SA, Ikeda M, Kito H, Akasaka N, Sasajima T and Sumpio BE: Endothelial cell response to different mechanical forces. J Vasc Surg 32: 789-794, 2000.

30. Oldenhof AD, Shynlova OP, Liu M, Langille BL and Lye SJ: Mitogen-activated protein kinases mediate stretch-induced c-fos mRNA expression in myometrial smooth muscle cells. Am J Physiol Cell Physiol 283: C1530-C1539, 2002.

31. Ferraro JT, Daneshmand M, Bizios R and Rizzo V: Depletion of plasma membrane cholesterol dampens hydrostatic pressure and shear stress-induced mechanotransduction pathways in osteoblast cultures. Am J Physiol Cell Physiol 286: C831-C839, 2004.

32. Kapur S, Baylink DJ and Lau KH: Fluid flow shear stress stimulates human osteoblast proliferation and differentiation through multiple interacting and competing signal transduction pathways. Bone 32: 241-251, 2003.

33. Schieker M, Pautke C, Haasters F, Schieker J, Docheva D, Böcker W, Guelkan H, Neth P, Jochum M and Mutschler W: Human mesenchymal stem cells at the single-cell level: simultaneous seven-colour immunofluorescence. J Anat 210: 592-599, 2007.

34. Barry FP and Murphy JM: Mesenchymal stem cells: clinical applications and biological characterization. Int J Biochem Cell Biol 36: 568-584, 2004.

35. Bobis S, Jarocha D and Majka M: Mesenchymal stem cells: characteristics and clinical applications. Folia Histochem Cytobiol 44: 215-214, 2007.

36. Mitchell DB, Santone KS and Acosta D: Evaluation of cytotoxicity in cultured cells by enzyme leakage. J Tissue Cult Methods 6: 113-116, 1980.

37. Gundberg C, Looker A, Nieman S and Calvo M: Patterns of osteocalcin and bone specific alkaline phosphatase by age, gender, and race or ethnicity. Bone 31: 703-708, 2002. 